TIPA. Travaux interdisciplinaires sur la parole et le langage

$36 \mid 2020$

Comment le corps coconstruit les discours et le sens

\title{
Comment le corps coconstruit les discours et le
} sens

Brahim Azaoui et Marion Tellier

\section{OpenEdition}

1 Journals

Édition électronique

URL : http://journals.openedition.org/tipa/4106

DOI : 10.4000/tipa.4106

ISSN : 2264-7082

Éditeur

Laboratoire Parole et Langage

Référence électronique

Brahim Azaoui et Marion Tellier, « Comment le corps coconstruit les discours et le sens », TIPA. Travaux interdisciplinaires sur la parole et le langage [En ligne], 36 | 2020, mis en ligne le 01 juin 2020, consulté le 26 janvier 2021. URL : http://journals.openedition.org/tipa/4106 ; DOI : https://doi.org/ 10.4000/tipa.4106

Ce document a été généré automatiquement le 26 janvier 2021.

a revue TIPA. Travaux interdisciplinaires sur la parole et le langage est mise à disposition selon les termes de la licence Creative Commons Attribution - Pas d'Utilisation Commerciale - Pas de Modification 4.0 International. 


\title{
Comment le corps coconstruit les discours et le sens
}

\author{
Brahim Azaoui et Marion Tellier
}

\section{Faire corps avec le langage}

1 Le corps, pris dans une acception large (regard, gestes manuels, proxémie et postures.), a récemment connu un regain d'intérêt dans divers domaines des sciences humaines. Depuis le tournant praxéologique qu'ont vécu les sciences du langage dans ces années 1950 avec les théories des actes du discours notamment, la linguistique interactionnelle (Kerbrat-Orecchioni, 2004; Mondada, 2004) lui consacre une certaine place dans ses travaux. Tout autant que les phénomènes verbaux et vocaux, il participe à la construction du sens auquel les interactants collaborent dans l'échange. Il convient donc de considérer à la suite de Kerbrat-Orecchioni (2004), Mondada (2004), Goodwin (2000), Kress (2015), Varela (Varela et al., 1991) ou McNeill (2005), entre autres chercheurs, que le discours ne peut se résumer à sa réalisation voco-verbale. Dans la logique qui prévaut dans une sémiotique sociale telle que définie dès les années 1990 par le New London Group (1996), auquel appartiennent des scientifiques tels que Kress, notamment, le langage est composé de tout ce qui fait signe et sens. Les travaux de Charles Goodwin (2000) confortent l'idée selon laquelle le corps constitue un signe au même titre que l'écriture, les images fixes, les symboles, etc. Soustraire un phénomène revient à amputer l'interaction d'une partie de sa signification. L'on retrouve cette même idée dans le paradigme de l'énaction, qui s'inspire principalement des travaux de Varela. Pour ce neurophénoménolgue chilien, le dualisme cartésien n'est pas recevable : il ne peut y avoir de distinction corps-esprit. Il existe un rapport sensorimoteur au monde, à l'environnement et aux autres qui implique de reconnaitre le langage comme étant constitué tout aussi bien des éléments verbaux que des phénomènes dits " non verbaux ", émotions incluses. Une réflexion similaire était déjà présente dans les travaux de Jousse en anthropologie du geste. Les Gesture studies, quant à elles, ont permis grâce aux études menées par McNeill (2005) ou Kendon (2004), de mettre au jour le lien indissociable qui existe entre gestes, parole et pensée. Que cela 
soit sur le plan cognitif, temporel ou sémantique, les gestes sont étroitement liés au discours verbal produit.

2 Le champ de la didactique a progressivement reconnu une place à la corporéité du langage dans le processus d'enseignement et d'apprentissage grâce notamment aux travaux précurseurs, en France, de Pujade-Renaud (1983), et, aux États-Unis, de Grant \& Grant Hennings (1971) ou encore de Balzer dès la fin des années 1960 (cité dans Beebe, 1980, p.4). L'impact des gestes et de leur reproduction dans le processus de mémorisation a été mis en évidence dans différentes études depuis les années 2000 (Macedonia et al., 2011; Tellier, 2008), dans l'apprentissage des sciences ou des langues (Aden, 2017 ; Alibali et al., 2011 ; Goldin-Meadow et al., 2009 ; McCafferty \& Stam, 2008 ; Sime, 2006 ; Tellier, 2014) et dans l'enseignement auprès d'élèves allophones (Hidden et al., 2020). Pour ce qui est de la dimension corporelle des relations interpersonnelles et dans l'acte pédagogique, de récentes études ont rappelé l'importance de l'interaction regard-gestes dans l'organisation multimodale des échanges simultanés et de la distribution des tours de parole (Azaoui, 2015 ; Mondada, 2009), le rôle de la proxémie et de la posture de l'enseignant dans le travail de différenciation (Azaoui, 2019 ; Forest, 2006 ; Moulin, 2004) et de bienveillance (Azaoui, 2021).

3 Pourtant, si ce champ d'étude que constitue la corporéité du langage, voire la multimodalité plus globalement, gagne en intérêt comme en témoignent les ouvrages publiés sur le sujet ces dernières années (Mazure-Palandre \& Colon de Carvajal, 2019 ; Rivière \& Blanc, 2019 ; Tellier \& Cadet, 2014), force est de constater que rares sont les revues françaises à lui avoir consacré un numéro.

\section{Présentation du numéro}

Ce numéro des TIPA se propose de participer à la compréhension et à la diffusion de cette thématique en croisant des contributions appartenant à des orientations théoriques différentes. Tous les articles proposent un éclairage sur la façon dont le corps contribue tant à la construction du discours et des interactions qu'à leur compréhension. En cela ils visent à répondre à la question suivante : comment le corps des participants à l'échange coconstruit-il les discours et le sens dans les discours didactiques? Le "discours didactique» sera entendu ici comme toute situation dans laquelle le discours des interlocuteurs se donne pour objectif premier ou non de faire savoir/ apprendre. Cette conception s'inspire des travaux de Moirand sur la notion de didacticité (1993), qui permettent de distinguer les discours dont l'intention première est didactique, comme l'essentiel de ceux produits dans les situations scolaires, et ceux qui ne sont pas didactiques mais qui relèvent d'une intention didactique. Dès lors, ces discours peuvent avoir lieu dans des contextes autres que celui de la classe, que cela soit dans le cadre d'interactions en face à face présentiel ou distanciel (en visioconférence par exemple) ou dans le cadre d'interactions asymétriques dans lesquelles un expert doit adapter son discours pour un non expert pour expliquer ou convaincre (médecin/patient, parent/enfant, professionnel/client, discours politique...).

5 En cohérence avec les positions actuelles, les contributions rassemblées dans ce numéro proposent des analyses qui considèrent la parole et le corps en constante interaction, l'étude et la compréhension de l'un explicitant le fonctionnement de 
l'autre. Elles témoignent par ailleurs de la diversité des approches théoriques évoquées plus haut et permettent de croiser les regards sur un objet commun.

\section{Présentations des articles}

6 Un ensemble d'articles porte sur la place du corps dans la co-construction du sens dans diverses situations d'enseignement-apprentissage d'une langue étrangère. Cet intérêt n'est pas un hasard. En effet, dans le contexte d'une interaction exolingue, les aspects posturo-mimo-gestuels ( $\mathrm{PMG}$ ) prennent une dimension particulière, notamment lorsque le verbal est défaillant (en compréhension comme en production). Les trois premiers articles se focalisent sur la gestuelle des apprenants de langue, leur recours aux ressources PMG lorsqu'ils s'expriment en langue-cible et la façon dont leurs interlocuteurs s'appuient sur ces ressources pour co-construire le sens. Tout d'abord, l'article de Saghie Sharifzadeh et Cédric Sarré sur les processus inférentiels dans l'interaction multimodale en L2, analyse les interactions entre apprenants et montre comment les interactants s'appuient sur les ressources PMG pour mener à bien l'interaction. Dans le même esprit, l'article de Loulou Kosmala explore la manière dont les (dis)fluences peuvent être négociées de manière multimodale lors d'activités collaboratives conjointes en tandem exolingue. La particularité de ce contexte dialogique est que l'un des interactants est apprenant de la langue et l'autre est un locuteur natif qui endosse le rôle d'expert linguistique. Enfin, la contribution de Kang ShinTae s'intéresse au contexte d'apprentissage du français en Corée. L'auteur montre comment l'enseignant, peu ou pas coréanophone, s'appuie sur la gestuelle de ses apprenants coréens pour résoudre des incompréhensions lexicales.

7 Du côté de l'enseignement des langues, Benjamin Holt propose une analyse du rôle des gestes dans les explications lexicales par visioconférence en montrant comment de futurs enseignants utilisent leur corps grâce à l'usage de la webcam pour véhiculer du sens dans l'enseignement en ligne. Can Denizci et Brahim Azaoui interrogent la notion de geste interactif à l'aune du contexte spécifique de la classe de langue en retenant pour exemples les échanges deux contextes d'enseignement du FLE-S en France et en Turquie.

8 Si ces cinq premiers articles s'inscrivent dans une perspective influencée principalement par les Gesture studies pour montrer le rôle fondamental des ressources PMG dans l'apprentissage comme dans l'enseignement d'une langue vivante, la contribution proposée par Sandrine Eschenauer relève du paradigme énactif du langage. L'auteure nous invite à réfléchir à l'intégration plus explicite du corps dans le cadre de l'apprentissage des langues vivantes à l'école, notamment à travers une approche artistique de l'enseignement.

9 Enfin, Isabel Colón de Carvajal, Louis Maritaud, Benoit Chalancon et Justine Lascar s'intéressent aux interactions en contexte médical et donnent à voir une autre forme de discours didactique. À travers une analyse conversationnelle multimodale, les auteurs analysent la manière dont s'organise la transmission d'informations cliniques lors de relèves infirmières.

10 Alors que nous finalisions l'édition de ce numéro, nous avons appris le décès de notre collègue Dominique Boutet, emporté par la Covid 19. Maitre de conférences HDR à l'université de Rouen, Dominique Boutet était un spécialiste reconnu dans le domaine des études gestuelles et de la langue des signes française. Il avait d'ailleurs fait partie du

TIPA. Travaux interdisciplinaires sur la parole et le langage, 36 | 2020 
comité scientifique de ce numéro. Très peinés par cette nouvelle et par la perte qu'elle entraine, tant sur le plan amical que professionnel, nous avons décidé de dédier ce numéro à la mémoire de Dominique Boutet.

Nous avons invité sa collègue et amie, Aliyah Morgenstern, à lui rendre hommage dans un article qui vient clore ce numéro des TIPA.

\section{BIBLIOGRAPHIE}

Aden, J. (2017) Langues et langage dans un paradigme enactif, Recherches en didactique des langues et des cultures, 14, 1, en ligne : https://journals.openedition.org/rdlc/1085.

Alibali, M. W., Nathan, M. J., \& Y. Fujimori (2011) Gestures in the mathematics classroom: What's the point? in N. Stein \& S. Raudenbush (eds) Developmental cognitive science goes to school, New York, NY.: Routledge, Taylor \& Francis, p. 219-234.

Azaoui, B., (2015) Fonctions pédagogiques et implications énonciatives de ressources professorales multimodales. Le cas de la bimanualité et de l'ubiquité coénonciative, Recherches en Didactique des Langues et Cultures, 12, 2, p. 225-253.

Azaoui, B. (2019) Analyse de la proxémie chez un enseignant de langues en lycée professionnel, in Mazur-Palande, A. \& I. Colon-Carvajal, (eds) Multimodalité du langage dans les interactions et l'acquisition, Grenoble: UGA éditions, p. 181-210.

Azaoui, B. (2021, sous presse) Ce que le corps de l'enseignant dit de la bienveillance, in MyreBisaillon, J. \& F. Torterat (eds) L'acquisition du langage aux premiers temps de la scolarisation. Comment concilier exigence scolaire et épanouissement de l'enfant ? Paris: L'Harmattan.

Beebe, S. A. (1980) The Role of Nonverbal Communication in Education: Research and Theoretical Perspectives, Paper presented at the annual meeting of the Speech Communication Association, November. $13-16^{\text {th }}$, New York, NY.

Forest, D. (2006) Analyse proxémique d'interactions didactiques, Thèse de doctorat non publiée, Université de Rennes 2.

Goldin-Meadow, S., Cook, S. W. \& Z. A. Mitchell (2009) Gesturing gives children new ideas about math, Psychological science, 20, 3, p. 267-272.

Goodwin, C. (2000) Action and embodiment within situated human interaction, Journal of pragmatics, 32, 10, p. 1489-1522.

Grant, B. M. \& D. Grant Hennings (1971) The Teacher moves. An analysis of non-verbal activity, New York, NY.: Teacher College Press.

Hidden, M.-O., Leclère, M. \& L. Uribe (2020) Conduites d'étayage dans une séquence de grammaire en UPE2A : les outils didactiques au coeur de la multimodalité des pratiques enseignantes, in Mendonça Dias, C., Azaoui, B. \& F. Chnane-Davin (eds) Allophonie. Inclusion et langues des enfants migrants à l'école, Limoges: Lambert Lucas, p. 217-236.

Kendon, A. (2004) Gesture. Visible action as utterance, Cambridge: Cambridge University Press. 
Kerbrat-Orecchioni, C. (2004) Que peut-on « faire » avec du dire ? Cahiers de Linguistique Française, 26, p. 27-43.

Kress, G. (2015) Applied Linguistics and a social semiotic account of multimodality, AILA Review, 28, p. 49-71.

New London Group (1996) A pedagogy of multiliteracies: designing social futures, Harvard Educational Review, 66, p. 60-92.

Macedonia, M., Müller, K., \& A. D. Friederici (2011) The impact of iconic gestures on foreign language word learning and its neural substrate, Human Brain Mapping, 32, 6, p. 982-998.

Mazure-Palandre \& I. Colon de Carvajal (eds.) (2019), Multimodalité du langage dans les interactions et l'acquisition, Grenoble: UGA éditions.

McCafferty, S. G. \& G. Stam (eds.) (2008) Gesture: Second language acquisition and classroom research, New York, NY.: Routledge.

McNeill, D. (2005) Gesture and thought, Chicago, IL.: University of Chicago Press.

Moirand, S. (1993) Autour de la notion de didacticité, Les Carnets du Cediscor, en ligne : http:// journals.openedition.org/cediscor/600.

Mondada, L. (2009) La production de l'intelligibilité de l'action : une approche multimodale des procédés de sélection des locuteurs dans les interactions en classe, Études de linguistique appliquée, 1,153 , p. 25-40.

Mondada, L. (2004) Temporalité, séquentialité et multimodalité au fondement de l'organisation de l'interaction : le pointage comme pratique de prise de tour, Cahiers de Linguistique Française, 26, p. 269-292.

Moulin, J.-F. (2004). Le discours silencieux du corps enseignant. La communication non verbale du maître dans les pratiques de classe, Carrefours de l'éducation, 17, 1, p. 142-159.

Rivière, V., \& N. Blanc (eds.) (2019) Observer la multimodalité en situations éducatives. Circulations entre recherche et formation, Lyon: ENS Éditions.

Pujade-Renaud, C. (1983/2005) Le corps de l'enseignant dans la classe, Paris: L'Harmattan.

Sime, D. (2006) What do learners make of teachers' gestures in the language classroom? in M. Gullberg (ed) Special issue on Gestures and SLA, International Review of Applied Linguistics, 44, 2, p. 211-230.

Tellier, M. \& L. Cadet (eds.) (2014) Le corps et la voix de l'enseignant : théorie et pratique, Paris: Éditions Maison des Langues.

Tellier, M. (2008) The effect of gestures on second language memorisation by young children, in Gullberg, M., \& K. de Bot (eds.) Special issue Gestures in language development, Gesture, 8, 2, p. 219-235.

Tellier, M. (2014) Donner du corps à son cours, in M. Tellier \& L. Cadet (eds.) Le corps et la voix de l'enseignant : théorie et pratique, Paris: Éditions Maison des Langues, p. 101-114.

Varela, F., Thompson, E. \& E. Rosch (1991) The Embodied Mind: Cognitive Science and Human Experience, Cambridge, MA.: MIT. 


\section{AUTEURS}

\section{BRAHIM AZAOUI}

LIRDEF, Univ de Montpellier, Univ Paul Valery Montpellier 3, Montpellier, France brahim.azaoui@umontpellier.fr

\section{MARION TELLIER}

Aix Marseille Univ, CNRS, LPL, Aix-en-Provence, France

marion.tellier@univ-amu.fr 